\title{
Kidney Rhabdoid Tumor
}

This neoplasm is also known as rhabdoid tumor of the kidney (RTK).

For details $\rightarrow$ see Chap. 144. 